\title{
Linguistic validation of cystic fibrosis quality of life questionnaires
}

\author{
Tatiana Rozov, ${ }^{1}$ Maristela T. Cunha, ${ }^{2}$ Oliver Nascimento, ${ }^{3}$ \\ Alexandra L. Quittner, ${ }^{4}$ José R. Jardim ${ }^{5}$
}

\begin{abstract}
Objective: The purpose of this study was to validate the Portuguese translations of four cystic fibrosis quality of life questionnaires (CFQ). The first three were developed for patients with cystic fibrosis aged from 6 to 11 years, from 12 to 13 years and 14 years or more, while the fourth was developed for the parents of patients aged 6 to 13 years.

Material and methods: The four CFQ translations contained from 35 to 50 questions covering nine domains and were validated as follows: translation from English to Portuguese, pilot application, back translation and then approval by the author of the English versions. The four translations were applied to 90 stable patients ( 30 from each age group) and the parents of patients aged 6-13 years $(n=60)$, on two occasions with a 13 to 17 day interval. Intraclass Correlation Coefficients (ICC) were used to measure reproducibility. This study was approved by the Commission for Ethics in Research at the institution.

Results: Reproducibility was good (ICC $=0.62$ to 0.99 ) for the four translations in all domains, with the exceptions of the Digestion domain for the 6 to 11 and 12 to 13 years age groups with ICC $=0.59$ and 0.47 , respectively and the Social Role domain for the 14 and over age group (ICC $=-0.19$ ).

Conclusion: The translation and cultural adaptation for Brazil resulted in four CFQ versions that are easy to understand and offer good reproducibility.
\end{abstract}

J Pediatr (Rio J). 2006;82(2):151-6: Questionnaires, quality of life, cystic fibrosis, validation.

\section{Introduction}

Mucoviscidosis - or cystic fibrosis (CF) or fibrocystic disease of the pancreas - is the most common lethal genetic diseases among Caucasians and is the most common fatal hereditary disease among industrialized

1. Livre-docente, Departamento de Pediatria, Faculdade de Medicina, Universidade de São Paulo (FMUSP), São Paulo, SP, Brasil. Professora, Universidade Federal de São Paulo, Escola Paulista de Medicina (UNIFESP/EPM), São Paulo, SP, Brasil. Médica colaboradora, Faculdade de Medicina do ABC (FMABC), Santo André, SP, Brasil.

2. Mestre, Centro de Reabilitação Pulmonar, UNIFESP/EPM, São Paulo, SP, Brasil. Fisioterapeuta, Instituto da Criança Professor Pedro de Alcântara, Hospital das Clínicas, Faculdade de Medicina, Universidade de São Paulo (HCFMUSP), São Paulo, SP, Brasil.

3. Médico. Vice-diretor, Centro de Reabilitação Pulmonar, UNIFESP/EPM, São Paulo, SP, Brasil.

4. Professor, Department of Psychology, University of Miami, Miami, USA.

5. Diretor, Centro de Reabilitação Pulmonar, UNIFESP/EPM, São Paulo, SP, Brasil. Professor adjunto, UNIFESP/EPM, São Paulo, SP. Coordenador, Programa de Pós-Graduação em Reabilitação Pulmonar, UNIFESP, São Paulo, SP, Brasil.

Manuscript received Jul 19 2005, accepted for publication Nov 092005.

Suggested citation: Rozov T, Cunha MT, Nascimento O, Quittner AL, Jardim JR. Linguistic validation of cystic fibrosis quality of life questionnaires. J Pediatr (Rio J). 2006;82:151-6. nations. Nevertheless, as a result of changes over the last two decades, with the increased understanding of this disease that has been gained since the CF gene was discovered, approximately $35 \%$ of those children who would have previously died, are nowadays surviving to adulthood. 1

More than 1,100 different CF gene mutations have been described since 1989 and the varying degrees of clinical manifestation severity depend on genotype and result in the obstructive phenomena observed in the exocrine glands, with very thick secretions. Cystic Fibrosis is characterized by chronic progressive suppurative obstructive lung disease; pancreatic insufficiency with maldigestion and malabsorption; secondary malnutrition; increased chlorine and sodium concentrations in sweat and male infertility in adulthood. ${ }^{2}$

Multiple organ dysfunctions and the long and complex daily treatment that is required have a significant impact on the quality of life (QoL) of these patients. Recently, emphasis has been placed on the concept that QoL assessments comprise a highly important additional clinical 
measure, both for the clinical study of CF and for monitoring individuals. ${ }^{3}$ Many disease-specific instruments and questionnaires have been developed over the last twenty years to assess QoL in CF, for both children and adults. ${ }^{4-9}$ Some of these questionnaires have been validated in several different languages over the last ten years. ${ }^{10-12}$

A suite of questionnaires originally developed and validated in France in 1996 are of most interest since they cover individuals from childhood to adulthood. ${ }^{13}$ These questionnaires were later translated into English and validated with a good level of consistency being observed ${ }^{10}$ plus the added advantage of being applicable to younger children. ${ }^{12}$ Recently, they have been validated in German, ${ }^{11}$ Spanish ${ }^{9}$ and Danish. ${ }^{14}$

It is estimated that there are $2000 \mathrm{CF}$ patients in Brazil and close to 3000 hospital admissions due to the disease have been recorded over the past five years. ${ }^{15}$ These patients are treated at several CF Centers and their treatments involve polysystemic care with many medications ingested and inhaled, involving nebulizations, antibiotics, pancreatic enzymes and nutritional supplements in addition to respiratory physiotherapy. These treatments consume both time and energy, are complex and often need to be repeated several times a day to achieve beneficial effects. Consequently, the health-related quality of life (HRQL) of these patients is far below the ideal, considering the WHO definition that health is a state of complete physical and social well being, and not just the absence of disease. Despite this, the HRQL of this population has not yet been assessed in Brazil because of the lack of reliable, internationally accepted and validated instruments.

The purpose of this study was to translate into Portuguese and validate a progressive series of four cystic fibrosis quality of life questionnaires (CFQ) in English. The first three were developed for patients with cystic fibrosis aged from 6 to 11 years, from 12 to 13 years and 14 years or more, while the fourth was developed for the parents of patients aged 6 to 13 years. ${ }^{16}$

\section{Material and methods Validation protocol}

The same steps were employed for cultural adaptation and validation for Brazil as have previously been described for the Saint George Respiratory Diseases Questionnaire ${ }^{17}$ and the Airways Questionnaire (AQ 20).18,19

The four CFQ versions contain from 35 to 50 questions each, depending upon age group (44 on the parents' version), covering nine QoL domains. The validation processes consisted of initial translation into Portuguese to produce a first version of each. A pilot study was then run in which these questionnaires were applied to $15 \mathrm{CF}$ patients (five for each age range) and to the parents of the ten patients aged 6-13 years, assessing doubtful or difficult parts of the text with emphasis on cultural equivalence. Based on these findings, a second version was produced of each questionnaire, on which certain demographic items had been adapted for Brazil. These questionnaires were then back translated into English by a Brazilian doctor who had not seen the CFQs before. These back translations were then compared to the English originals and the author of those originals analyzed them and gave her approval. The four final CFQ versions were then produced in Portuguese and named $\mathrm{CFQ}_{6-11}, \mathrm{CFQ}_{12-13} \mathrm{CFQ}_{14+}$ and $\mathrm{CFQ}_{\text {Parents11-13 }}$ (the full texts of the questionnaires are available online at www.jped.com.br/ing).

\section{Patients}

The four translations were then applied to $90 \mathrm{CF}$ patients (30 in each age group) and to the parents of the $60 \mathrm{CF}$ patients aged 6-13 years.

\section{Inclusion criteria}

I - Diagnosis of CF according to classical criteria; ${ }^{20}$ II - Patients above 6 years of age; III - Clinically stable before and during the study, confirmed by means of a clinical stability questionnaire; IV - Consent to take part in the study.

\section{Exclusion criteria}

I - Failure to completely fill out or to understand the questionnaires; II - Refusal by patients or guardians to sign free and informed consent.

\section{Clinical stability questionnaire}

This questionnaire comprised eight questions about clinical occurrences during the thirty days prior to the first interview and the fifteen days prior to the second: fever during the previous week, increase in coughing and shortness of breath, increase in and changes to the characteristics of expectoration, requirement of and/or increase in oxygen use, emergency care and/or hospitalization, increase in medication and antibiotic use beyond the habitual.

\section{Structure and application of the questionnaires}

The 4 CFQ translations contain 35 questions for age groups from 6 to 13 years, 50 questions for 14 year-olds and over and 44 for parents, covering nine QoL domains, 3 symptom scales and one item related to health perception: Physical, Body Image, Emotion, Social/School, Social Role, Vitality, Eating, Treatment Burden, Digestion, Respiratory, Weight and Health. An interviewer filled out questionnaires for patients from 6 to 11 years using 
flashcards to help the children choose their answers. Older patients filled out their own questionnaires (selfassessment). The parents of 6 to 13 -year-old patients filled out the questionnaires themselves and care was taken to ensure that parents and patients did not have a mutual influence on each other's answers.

Reproducibility was assessed by applying the questionnaires to the same patients on two different occasions with an average interval of fifteen days (plus or minus two days) with the same interviewer conducting both sessions. There were eight interviewers who had been previously trained to clear up doubts and to read questionnaires aloud to illiterate subjects and children from 6 to 11 with a neutral attitude to the answers.

\section{Medical ethics}

The research project was approved by the Committee for Ethics in Medical Research at Hospital São Paulo, Universidade de Federal de São Paulo (UNIFESP). Patients and/or caregivers signed a free and informed consent form.

\section{Statistical analysis}

The number of patients required for each CFQ version was calculated based on the past experience of the research coordinator with validating questionnaires for chronic obstructive pulmonary disease patients and on an estimation that the variation in responses to the questionnaires would be similar to published descriptions $(n=30) \cdot{ }^{17-19}$ Descriptive statistical analysis was employed for clinical and demographic characteristics. Reproducibility was measured using intraclass correlation coefficients (ICC) with confidence intervals of $95 \%(95 \% \mathrm{CI})$. The level of statistical significance was set at $p<0.05$.

\section{Results}

This was a multicenter sample with patients recruited at three CF Centers in São Paulo city and one center in each of the cities of Campinas, Ribeirão Preto, Botucatu, Belo Horizonte and Curitiba, in order to reach the necessary number of participants.

The principal characteristics of the 90 patients who completed the study are shown in Table 1.

The ICC scores for analyzing reproducibility over an average interval of 15 days were as follows: ICC $=0.90$ (95\%CI $0.84-0.95$ ) for the group of children aged 6 to 11 years; ICC $=0.84(95 \%$ CI $0.75-0.91)$ for 12 to 13 yearold group; and ICC $=0.92(95 \%$ CI $0.87-0.95)$ for 14 year-olds and above. For the group of parents of $\mathrm{CF}$ patients with CF aged 6-11 years and $12-13$ years coefficients for reproducibility were ICC $=0.91(95 \% \mathrm{CI}$ $0.85-0.95)$ and ICC $=0.92(95 \%$ CI $0.87-0.95)$, respectively (Table 2).

Reproducibility was good for all domains (ICC $=0.62$ to 0.99 ) for all 4 translations, with the exception of the Digestion domain (ICC $=0.59_{\mathrm{CFQ} 6-11}$ and $\mathrm{ICC}=$ $0.47_{\mathrm{CFQ} 12-13}$ ), and the Social Role domain (ICC $=$ $\left.-0.19_{\text {CFQ }>14}\right)$. The reproducibility of the questionnaire for children was also good for all domains, with the exception of Body Image with ICC $=0.50_{\text {CFQ6-11 }}$.

Table 1 - Demographic characteristics (percentages) of the 90 patients with cystic fibrosis who responded to the disease-specific questionnaires (CFQ)

\begin{tabular}{llccc}
\hline Age group & & $\mathbf{6 - 1 1}$ years & $\mathbf{1 2 - 1 3}$ years & > 14 years \\
\hline No. & & 30 & 30 & 30 \\
Sex & male & $20(66.7)$ & $10(33.3)$ & $16(53.3)$ \\
& female & $10(33.3)$ & $20(66.7)$ & $14(46.7)$ \\
Race & white & $26(86.7)$ & $20(66.7)$ & $26(86.7)$ \\
& mixed race & $4(13.3)$ & $4(13.3)$ & $1(3.3)$ \\
& black & - & - & $2(6.7)$ \\
& Native Brazilian Indians & - & $1(3.3)$ & $1(3.3)$ \\
& others & - & $2(6.7)$ & - \\
Age $(X \pm S D)$ & unsure & - & $3(10.0)$ & - \\
& & $8.9 \pm 1.9$ & $12.5 \pm 0.5$ & $21.4 \pm 6.7$ \\
\hline
\end{tabular}

$C F Q=$ cystic fibrosis quality of life questionnaire. $\mathrm{X} \pm \mathrm{SD}=$ mean \pm standard deviation 
Table 2 - Reproducibility (intraclass correlation coefficient) of domains of the four

\begin{tabular}{|c|c|c|c|c|c|}
\hline Domains & $\mathrm{CFQ}_{6-11}$ & $\mathrm{CFQ}_{12-13}$ & $\mathrm{CFQ}_{>14}$ & Parents 6-11 & Parents $_{12-13}$ \\
\hline Physical & $0.88 *$ & $0.89 *$ & $0.99 *$ & $0.94 *$ & $0.90 *$ \\
\hline Body image & $0.86 *$ & $0.78 *$ & $0.93 *$ & $0.50^{+}$ & $0.92 *$ \\
\hline Digestive & $0.59^{+}$ & $0.47^{\dagger}$ & $0.74 *$ & $0.78 *$ & $0.86 *$ \\
\hline Respiratory & $0.81 *$ & $0.88 *$ & $0.85 *$ & $0.83 *$ & $0.82 *$ \\
\hline Emotional & $0.80 *$ & $0.93 *$ & $0.80^{\ddagger}$ & $0.74 *$ & $0.82 *$ \\
\hline Social & $0.66^{\ddagger}$ & $0.81 *$ & $0.92 *$ & $0.62 \neq$ & $0.77 *$ \\
\hline Nutrition & $0.78 *$ & $0.81 *$ & $0.90 *$ & $0.84 *$ & $0.91 *$ \\
\hline Treatment & $0.78 *$ & $0.76 *$ & $0.82 *$ & $0.90 *$ & $0.93 *$ \\
\hline Vitality & - & - & $0.87 *$ & $0.82 *$ & $0.86 *$ \\
\hline Health & - & - & $0.83 *$ & $0.67^{\ddagger}$ & $0.68^{\neq}$ \\
\hline Social role & - & - & $-0.19 \S$ & - & - \\
\hline Weight & - & - & $0.81 *$ & $0.67^{\ddagger}$ & $0.81 *$ \\
\hline Total & $0.90 *$ & $0.84 *$ & $0.92 *$ & $0.91 *$ & $0.92 *$ \\
\hline
\end{tabular}

$\mathrm{CFQ}=$ cystic fibrosis quality of life questionnaire.

Level of significance of intraclass correlation coefficient: ${ }^{*} p<0.001 ;{ }^{\dagger} p<0.05 ;{ }^{\ddagger} p<0.01$; $\S$ not significant.

The mean times taken to complete the questionnaires at the first and second interviews respectively were $13.1 \pm 7.2$ and $9.4 \pm 3.6(p=0.005)_{C F Q 6-11} ; 11.4 \pm 4.0$ and $9.2 \pm 4.7(p=0.002)_{C F Q 12-13} ;$ and $13.9 \pm 4.7$ and $10.9 \pm 4.1$ $(p<0.001)_{C F Q 14+}$. Parents took longer to respond, with mean times of $16.1 \pm 8.6$ and $11.9 \pm 4.2(p=0.02)_{C F Q}$

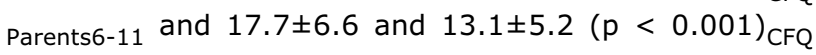
Parents12-13.

\section{Discussion}

The current concern with offering patients improvements in HRQL has stimulated researchers to investigate and quantify HRQL using QoL questionnaires. 21,22

Many generic questionnaires have been used for $\mathrm{CF}$, such as the Quality of Well Being, Nottingham Health Profile, Sickness Impact Profile, Self Administered Dependency Questionnaire, Functional Status, SF36 and other systems. ${ }^{23}$ Nevertheless, it is disease-specific questionnaires that have generated most interest among professionals in the area because they directly measure the physical, emotional and social impact of CF and its treatments on patients and their families. In order that HRQL can be quantified in countries with cultures and languages different from the country where questionnaires have been produced, it is recommended that translated instruments be validated and adapted for the conditions in the country where they are to be used. ${ }^{10-14}$ This is the reason why it was necessary to validate Portuguese translations of the CFQs since there were previously no QoL questionnaires for CF patients published in Brazil. The choice of the four-part CFQ for translation and validation was due to its progressive and wide-ranging character (from 6 years to adulthood), the ease of application and the ability to quantify numerically and over time the changes that happen to each patient within each domain.

ICC is the adequate test to evaluate reproducibility, with values equal or above $0.7^{24}$ and for CFQs the acceptable level is 0.6 or above. ${ }^{16}$ During the validation process we observed that, with the exception of two domains, the Digestion for age groups from 6 to 13 years and the Social Role domain for patients 14 years and over, all other domains had ICC values over 0.6. It is interesting to observe that patients over 14 years old were unable to provide reproducible responses over the short term relating their social functioning and inclusion, i.e. their roles within the environment in which they live, perhaps because they had never considered this type of personal questioning.

When we compared our data with those obtained by Quittner et al. ${ }^{16}$ we found higher ICC values in 39/45 $(86.7 \%)$ of possible comparisons, lower values in 5/45 $(11.1 \%)$ and one coefficient that was equal for both datasets $(2.2 \%)$, which suggests that the instrument we have validated has good reproducibility, under the conditions in which it was applied and at a fifteen-day interval. The English versions of these CFQs had lower reproducibility scores. We consider these results to be 
highly relevant, bearing in mind the cystic fibrosis population we studied is not used to answering QoL questionnaires, in contrast with the CF population in the United States. An important factor in the good results is the fact that the majority of interviewers already had experience with questionnaires. Another aspect of fundamental importance to the level of reproducibility obtained was the prior training given to all team members specifically for this situation, making the process of questionnaire application very homogenous. Interviews should preferably always be undertaken by the same interviewer to avoid distortions, particularly with younger patients. We observed that the participants learned to respond to the questionnaires with significant reductions in the mean time taken to reply on the second occasion, for all groups including the 6 to 11 year-olds who are the hardest work. As a result of this research we can conclude that translation and adaptation to the Brazilian language and culture produced four translated CFQs that proved easy to understand and offer good reproducibility.

With respect to the applicability of questionnaires, just 16 studies over the last 15 years had QoL as an outcome and none of them offer conclusive results on the QoL of CF patients. ${ }^{25}$ The authors of this review point out that even in well designed clinical trials it is necessary to justify the rationale for measuring QoL, to define the QoL measurement instrument adequately, to choose a scale (or score) that is sufficiently sensitive to detect minimal changes in QoL, to calculate sample size, to describe the statistical methods and analyses used, to discuss the clinical importance in changes to QoL and to evaluate the methodological quality of the research with respect to QoL. Validated questionnaires, disease-specific scales, rational construction of domains and study of patients' baseline QoL patterns are obligatory initial elements. If data is lost during the study (because not recorded or because patients die) and if the confidence intervals of variables studied are not provided, it becomes difficult to assess clinical QoL results. The validity, reliability and sensitivity of the instruments employed (questionnaires) are of fundamental importance to obtaining high quality evidence. 25

In conclusion, the translated and culturally adapted Brazilian versions of the CFQs demonstrated ease of understanding and good reproducibility.

\section{Acknowledgements}

Thanks are due to the coordinators of the research centers for their time and support and for making it possible for their patients to take part in the study:

- Dr. Neiva Damaceno at the Cystic Fibrosis Treatment Center, Pediatrics Department, Santa Casa de Misericórdia de São Paulo;
- Dr. Sonia Chiba and Dr. Clóvis T. Gomes at the Pediatric Pneumology Clinic, Escola Paulista de Medicina (UNIFESP);

- Dr. Antonio F. Ribeiro and Dr. José D. Ribeiro at the Pediatrics Department, Medical Sciences Faculty, Hospital das Clínicas, Universidade Estadual de Campinas (UNICAMP);

- Dr. Joaquim C. Rodrigues and Dr. Fabíola V. Adde at the Pneumology Unit, Children's Institute, Hospital das Clínicas, Universidade de São Paulo (FMUSP);

- Dr. Francisco C. Reis and Dr. Alberto Avergara at the Pneumology Clinic, Centro Geral de Pediatria (CGP), Belo Horizonte;

- Dr Carlos Ried from the Hospital Pequeno Príncipe clinic and also the Associação Brasileira de Assistência a Mucoviscidose (ABRAM), Curitiba;

- Dr. Giesela F. Ferrari from the Pediatric Pneumology Clinic at the Botucatu Medical Faculty, Universidade Estadual Paulista (UNESP);

- Dr. Lídia G. M. M. Torres at the Pediatrics Department, Hospital das Clínicas, FMUSP, Ribeirão Preto.

We are also grateful to ROCHE Químicos e Farmacêuticos for the logistic and financial support and for the initial statistical analysis.

Finally, thanks go to Dr. Lucília Santana Faria at the Children's Institute, Hospital das Clínicas, FMUSP for back translating the CFQs from Portuguese into English.

The full versions of the four cystic fibrosis quality of life questionnaires are available online at www.jped.com.br/ing.

\section{References}

1. Cystic Fibrosis Foundation. Patient registry 2001 annual data report. Bethesda: CF Foundation; 2002.

2. Orenstein DM, Winnie GB, Altman H. Cystic fibrosis: a 2002 update. J Pediatr. 2002;140:156-64.

3. Quittner AL. Measurement of quality of life in cystic fibrosis. Curr Opin Pulm Med. 1998;4:326-31.

4. Czyzewski DI, Mariotto MJ, Bartholomeu LK, Le Compte SH, Sockrider MM. Measurement of quality of well being in a child and adolescent cystic fibrosis population. Med Care. 1994;32:965-72.

5. Staab D, Wenninger K, Gebert N, Rupprath K, Bisson S, Trettin $M$, et al. Quality of life in patients with cystic fibrosis and their parents: what is important besides disease severity? Thorax. 1998; 53:727-31.

6. Bradley J, Dempster M, Wallace E, Elborn S. The adaptations of a quality of life questionnaire for routine use in clinical practice: the Chronic Respiratory Disease Questionnaire in cystic fibrosis. Qual Life Res. 1999;8:65-71.

7. Gee L, Abbot J, Conway SP, Etherington C, Webb AK. Development of disease specific heath related quality of life measure for adults and adolescents with cystic fibrosis. Thorax. 2000;55: 946-54.

8. Goldbeck L, Schmitz TG. Comparison of three generic questionnaires measuring quality of life in adolescents and adults with cystic fibrosis: the 36 -item short form health survey, the quality of life profile for chronic disease, and the questions on life satisfaction. Qual Life Res. 2001;10:23-36. 
9. Henry B, Aussage P, Grosskopf C, Goehrs JM. Development of a cystic fibrosis questionnaire (CFQ) for assessing quality of life in pediatric and adult patients. Qual Life Res. 2003;12:63-76.

10. Quittner AL, Sweeny S, Watrous M, Munzenberger P, Bearss $K$, Nitza AG, et al. Translation and linguistic validation of a diseasespecific quality of life measure for cystic fibrosis. J Pediatr Psychol. 2000;25:403-14.

11. Wenninger K, Aussage $P$, Wahn U, Staab D, the German CFQ Study Group. The revised German cystic fibrosis questionnaire: validation of disease - specific health-related quality of life instrument. Qual Life Res. 2003;12:77-85.

12. Modi AC, Quitner AL. Validation of disease-specific measure of health-related quality of life for children with cystic fibrosis. J Pediatr Psychol. 2003;28:535-46.

13. Henry G, Grosskopf C, Aussage P, the CFQoL Study Group. Construction of a disease-specific quality of life questionnaire for cystic fibrosis. Pediatr Pulmonol. 1996;14(suppl):337-8.

14. Klinj PH, van Stel HF, Quittner AL, van der Net J, Doeleman W, van der Schans $C P$, et al. Validation of the Dutch cystic fibrosis questionnaire (CFQ) in adolescents and adults. J Cyst Fibros. $2004 ; 3: 29-36$.

15. Sistema de Informações Ambulatoriais do SUS. http:// w 3.datasus.gov.br/datasus/datasus.php?area $=$ 361A3B366C1D463E2F366G10HIJd3L1M0N\&VInclude $=$. . / site/ din_sist.php\&VSis=1\&VAba=0\&VCoit=463. Access: $10 / 07 / 2005$.

16. Quittner AL, Buu A, Warrous $M$, Davis MA. CFQ cystic fibrosis questionnaire. A health-related quality of life measure [English version 1.0 and 2.0]. Bethesda: CF Foundation; 2000.

17. Souza TC, Jardim JRB, Jones P. Validação do questionário do Hospital Saint George na doença respiratória (SGRQ) em pacientes portadores de DPOC no Brasil. J Pneumologia. 2000;16:119-25
18. Camelier A, Rosa $F$, Jones $P$, Jardim JR. Validation of the Airways Questionnaire 20 - AQ20 in patients with chronic obstructive pulmonary disease (COPD) in Brazil. J Pneumologia. 2003;29: 28-35.

19. Camelier A, Rosa F, Jones P, Jardim JR. Brazilian version of Airways Questionnaire 20: a reproducibility study and correlations in patients with COPD. Respir Med. 2005;99:602-8.

20. Rosenstein BJ, Cutting GR. The diagnosis of cystic fibrosis: a consensus statement. J Pediatr. 1998;132:589-95.

21. Abbott J, Gee L. Quality of life in children and adolescents with cystic fibrosis: implications for optimizing treatments and clinical trial design. Paediatr Drugs. 2003;5:41-56.

22. Clarke AS, Eiser $C$. The measurement of health-related quality of life (QOL) in paediatric clinical trials: a systematic review. Health Qual Life Outcomes. 2004;2:66.

23. American Thoracic Society. Quality of life. Specific diseases. Cystic fibrosis. www.atsqol.org/cystic.asp. Access: 17/05/2005.

24. Laureau S, Breslin EH, Meek PM. Functional status instruments: outcome measure in the evaluation of patients with chronic obstructive pulmonary disease. Heart Lung. 1996;25:212-24.

25. Abbott J, Hart A. Measuring and reporting quality of life outcomes in clinical trials in cystic fibrosis: a critical review. Health Qual Life Outcomes. 2005;3:19.

Correspondence:

José R. Jardim

Universidade Federal de São Paulo (UNIFESP)

Rua Botucatu, 740, $3^{\circ}$ andar

CEP 04023-062 - São Paulo, SP - Brazil

Fax: +55 (11) 5575.5035

E-mail: joserjardim@yahoo.com.br 